\title{
Better clinical benefits and potential cost saving of an enhanced recovery pathways for laparoscopic adrenalectomy
}

\author{
Yue Yan ${ }^{1,2 \wedge}$, Jie Cheng ${ }^{1}$, Kang Chen ${ }^{1}$, Ting-Fang Liu ${ }^{2}$, Guang Ning ${ }^{1,2,3}$ \\ ${ }^{1}$ Dean's Office, Ruijin Hospital, Shanghai Jiao Tong University School of Medicine, Shanghai, China; ${ }^{2}$ Institute for Hospital Management, Tsinghua \\ University, Tsinghua Shenzhen International Graduate School, Beijing, China; ${ }^{3}$ Department of Endocrine and Metabolic Diseases, Ruijin Hospital, \\ Shanghai Jiao Tong University School of Medicine, Shanghai, China \\ Contributions: (I) Conception and design: Y Yan, TF Liu; (II) Administrative support: J Cheng, K Chen; (III) Provision of study materials or patients: \\ G Ning; (IV) Collection and assembly of data: Y Yan, J Cheng, K Chen; (V) Data analysis and interpretation: Y Yan; (VI) Manuscript writing: All \\ authors; (VII) Final approval of manuscript: All authors. \\ Correspondence to: Ting-Fang Liu, PhD. Institute for Hospital Management, Tsinghua University, No. 30 Shuangqing Road, Haidian District, Beijing \\ 100080, China. Email: liutf@mail.tsinghua.edu.cn; Guang Ning, MD, PhD. Department of Endocrine and Metabolic Diseases, Ruijin Hospital, \\ Shanghai Jiao Tong University School of Medicine, Shanghai Institution of Endocrine and Metabolism Diseases, No. 197 Ruijin Road No. 2 , \\ Shanghai 200025, China. Email: guangning@medmail.com.cn.
}

Backgroundk Enhanced recovery pathways (ERPs) have been demonstrated to be clinically and economically effective for colorectal surgery. However, data are lacking to support the cost-effectiveness of ERPs for laparoscopic adrenalectomy, as well as potential economic loss. This study aims to determine the cost-effectiveness of ERPs vs. conventional care (CC) for patients undergoing laparoscopic adrenalectomy.

Methods: A single centered retrospective case-control study was performed. Patients undergoing laparoscopic adrenalectomy from January 2018 to June 2021 were enrolled. One inpatient ward used ERPs, whereas the other did not. Case control was used for matching (53 patients for each group). Postoperative outcomes were recorded up to 30 days after surgery. ERPs-specific costs were integrated into the model. Preoperative, intraoperative and postoperative real costs were collected for each patient via the hospital administration, which were reported in Renminbi. A bootstrap independent $t$-test was used for comparison.

Results: The two groups were matched in terms of demographics and surgical details. Hospital stay was significantly shorter in the ERPs group (2.54 days, $\mathrm{P}<0.001)$. The rate of complications was lower in the ERPs group ( 1 vs. 7 patients, $\mathrm{P}=0.031$ ), there was no severe complications or morbidity. ERPs-specific costs were $¥ 656$ per patient. The self-payment cost difference per patient between the two groups was $¥ 3,154.52$ in favor of the ERPs group, but this was not statistically significant $(\mathrm{P}=0.113)$. The mean direct saving per patient in the ERPs group was $¥ 8,326.00$, the total costs saving including indirect and potential overhead per patient was $¥ 18,247.60$.

Conclusions: Enhanced recovery is cost-effective compared with conventional perioperative management for laparoscopic adrenalectomy. Savings were noted in disposable materials, medicine and medical services, radiology and laboratory costs, with no increase in morbidity or duration.

Keywords: Laparoscopic adrenalectomy; cost-effectiveness; enhanced recovery pathway (ERP); enhanced recovery after surgery (ERAS); day surgery

Submitted Jul 30, 2021. Accepted for publication Dec 18, 2021.

doi: $10.21037 /$ gs-21-504

View this article at: https://dx.doi.org/10.21037/gs-21-504

^ ORCID: 0000-0001-9434-4223. 


\section{Introduction}

Adrenal tumors can be categorized as either functional (hormone-secreting) or silent (either benign or malignant). Laparoscopic adrenalectomy remains the mainstay of curative therapy for adrenal mass (1). Overall, less than $1 \%$ of these tumors are cancerous while the surgery is still imposing a burden on both society and family $(2,3)$.

Enhanced recovery pathways (ERPs) have been shown to decrease hospitalization and complications after colorectal surgery (4), and have been demonstrated to improve clinical outcomes in almost all major surgical specialties, like gastrectomy (5), and neurosurgical (6), amongst others. ERPs have been progressively implemented in urology and the guidance for bladder cancer has been available since $2013(7,8)$. The experience in care and surgical disciplines encourages the development of an ERP for radical cystectomy.

It reported ERPs in clinical practice led to a significant reduction in time to first flatus, to stool and to normal diet. Also, readmission rate and complication rate were lower in ERP group compared with traditional care management (9).

And the better recovery outcomes have also been proved in China (10). Patients that experienced nephrectomy, low complication rates and reduced hospital stay were reported in 45 patients managed by ERPs.

With the trans-European surgery experience uniformly showing effectiveness with implementation of enhanced recovery after surgery (ERAS), the ERPs may need more practice and reassessment in different regions and ethnicities $(7,11,12)$. Hospital managers have been aware of the promising results of ERAS, but ERPs have yet to be widely adopted in China. Adoption of ERPs has been difficult to fully implement for its numerous recommendations (13). As health care spending continues to grow, the burden of public medical treatment is gradually reduced (14), the cost savings associated with ERPs may further encourage the adoption in public hospitals.

Furthermore, few studies comparing ERPs with conventional perioperative management for patients undergoing laparoscopic adrenalectomy have investigated implementation and maintenance costs, post-discharge patient-reported outcomes, and socioeconomic impact (15), which may significantly affect the cost-effectiveness of ERPs $(16,17)$. Before widespread, a formal economic evaluation assessing both the costs and benefits of these pathways is required to determine the cost-effectiveness. The main objective of this study, therefore, was to investigate the cost-effectiveness of ERPs $v s$. conventional perioperative management of patients undergoing laparoscopic adrenalectomy. We present the following article in accordance with the TREND reporting checklist (available at https:// gs.amegroups.com/article/view/10.21037/gs-21-504/rc).

\section{Methods}

\section{Patients}

All patients undergoing laparoscopic adrenalectomy for adrenal tumor at Ruijin Hospital (Shanghai, China) between January 2018 and June 2021 were retrospectively recruited. Patients were divided into conventional care (CC) and ERP groups. Inclusion and exclusion criteria were similar for both groups. Patients who underwent simultaneous nephrectomy or robotic surgery, or who were diagnosed with Cushing's syndrome or pheochromocytoma, were excluded for the benefit bias outside of pathway factors. The study was a retrospective matched casecontrol design, evaluating the association between ERPs and outcomes variables (both clinically and economically). Five controls were recruited for each case and matched based on the location of the tumor (left or right), diagnosis (functional or nonfunctional), age group (error within 15 years), tumor size (error within $1 \mathrm{~cm}$ ), body mass index (BMI) (error within $3.5 \mathrm{~kg} / \mathrm{cm}^{2}$ ) and sex (male, female). Researchers wanted to ensure that the control group would have the same single and multiple tumor diversity as the cases. Given that the control group consisted of a higher proportion of single tumor than multiple tumors, additional matching based on the number of tumors ensured that the controls selected would not be biased based on the sampling frame (Figure 1). The groups were well matched in terms of demographic and tumor details. Patients with primary aldosteronism were rarely but included.

There are two wards for the department of urology, one of which focuses on day surgery. Consecutive patients treated during implementation of the ERPs in the day ward were compared with those treated in the other ward without its introduction. Starting in January 2018, patients in the day ward were enrolled in a multidisciplinary ERP incorporating preoperative counselling, reduced preoperative fasting, preoperative carbohydrate loading, avoidance of premedication, optimized fluid balance, standardized postoperative analgesia, use of a no-drain policy (18), as well as early nutrition and mobilization (Table 1). The care measures were delivered by an integrated team of surgeon, physiotherapist, nurse, anesthetist and dietician. 


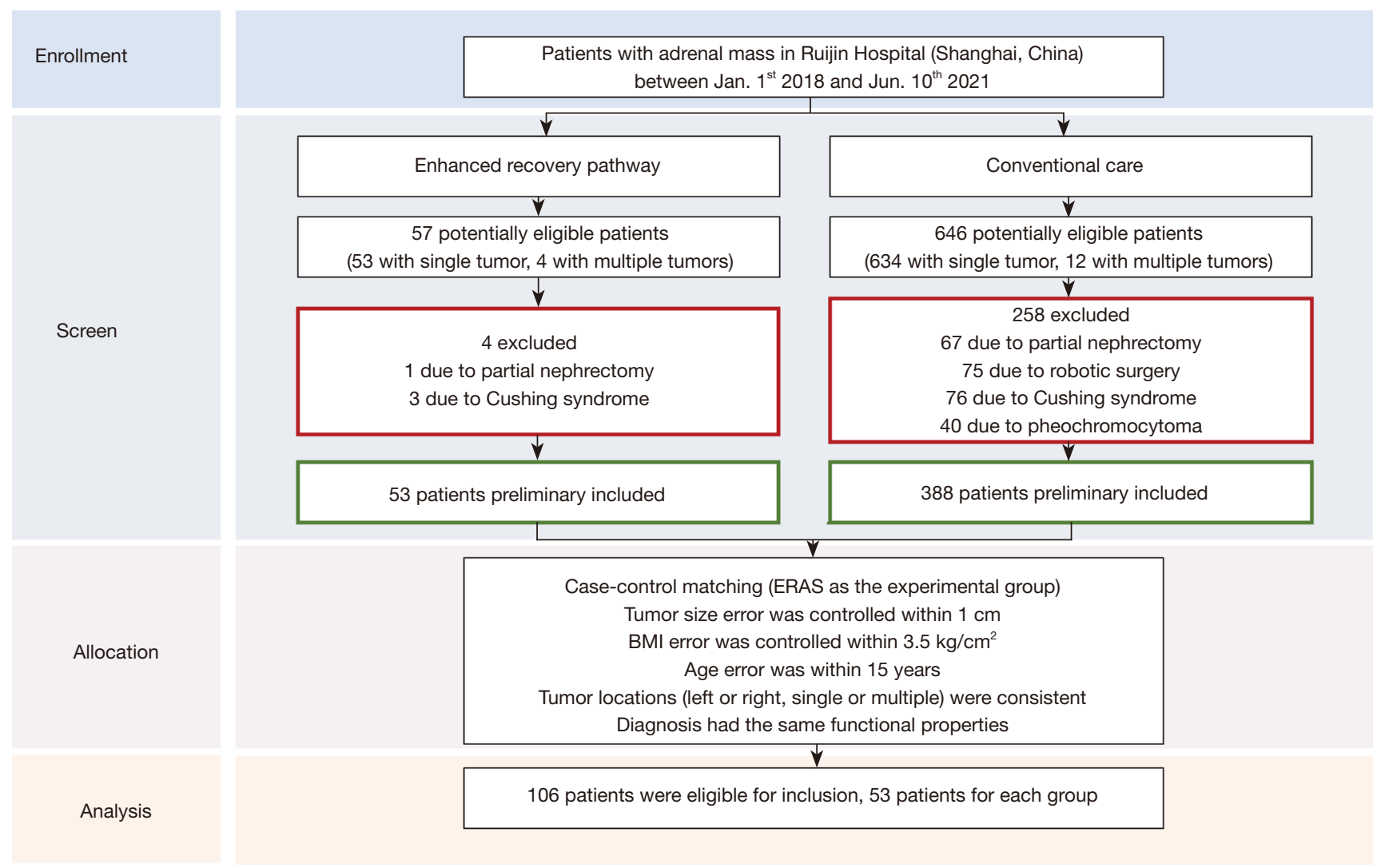

Figure 1 Study flowchart. Cases and controls in study design. Shown is the chronologic sequence of events for patients who were included in the case-control study. There was no overlap between the cases and controls, since some of the patients were admitted to the day ward, patients undergoing laparoscopic adrenalectomy were divided into two different groups, receiving multidisciplinary enhanced care and CC, respectively. Patients diagnosed as multiple (or single) tumors were controlled with multiple (or single) tumors samples, and other five matching conditions must be met within a certain range (tumor size, BMI, age, tumor locations, function). A strict case-control ratio of 1:1 was selected to maximize the statistical power of the study. CC, conventional care; BMI, body mass index; ERAS, enhanced recovery after surgery.

All surgeries were performed by a standing team consisting of three surgeons led by a senior surgeon with extensive experience in laparoscopic surgery (all beyond their learning curve), and the operation has been standardized over the years into a safe and reproducible procedure. The study was conducted in accordance with the Declaration of Helsinki (as revised in 2013). The study was approved by the Ethics Committee of the Ruijin Hospital, Shanghai Jiao Tong University School of Medicine (No. 2020-00648), and informed consent was taken from all individual participants.

\section{Clinical outcomes and criteria}

Clinical outcomes were recorded for up to 30 days after surgery. The comorbidity status was assessed, taking into account the malnutrition state and severity of disease.
Comorbidity was classified using the age-adjusted Charlson comorbidity score, a modified combined comorbidity index $(19,20)$. Length of stay (LOS) data included duration of primary hospital admission as well as any readmissions within 30 days after surgery. Complications were recorded in number and graded according to the Dindo-Clavien classification (21).

The following discharge criteria were used in the ERPs group: pain control with oral analgesia, passage of flatus or feces, completion of the tube drawing, and independent ambulation. Patients in the control group received standard postoperative care and were discharged from hospital at the surgeon's discretion.

\section{Cost analysis}

Medical costs were calculated by micro costing, in which the 
Table 1 Comparison of conventional and enhanced recovery perioperative management for adrenal tumors

\begin{tabular}{|c|c|c|c|}
\hline Phases & Measures & CC group $(n=53)$ & ERP group $(n=53)$ \\
\hline \multirow{5}{*}{$\begin{array}{l}\text { Preoperative } \\
\text { management }\end{array}$} & $\begin{array}{l}\text { Counselling and } \\
\text { education }\end{array}$ & Oral patient education by nurses & $\begin{array}{l}\text { Psychological preparation for surgery and } \\
\text { postoperative recovery: oral and written publicity } \\
\text { pamphlets of perioperative pathway, daily diet and } \\
\text { ambulation plan, expected discharge date }\end{array}$ \\
\hline & Exercise instructions & $\begin{array}{l}\text { No instructions for preoperative exercise } \\
\text { given }\end{array}$ & $\begin{array}{l}\text { Preoperative exercise optimization including respiratory } \\
\text { muscle strengthening and breathing exercises }\end{array}$ \\
\hline & Fasting & Fasting starting midnight before surgery & $\begin{array}{l}\text { Clear fluids allowed until } 4 \mathrm{~h} \text { before surgery, solids } \\
\text { until } 8 \mathrm{~h} \text { before surgery }\end{array}$ \\
\hline & Carbohydrate drinks & No carbohydrate drinks & $\begin{array}{l}800 \mathrm{~mL} \text { on evening and } 400 \mathrm{~mL} 2 \text { hours before } \\
\text { surgery }\end{array}$ \\
\hline & Thromboprophylaxis & $\begin{array}{l}\text { LMW heparin } 12 \text { hours before surgery and } \\
\text { during } 4 \text { weeks }\end{array}$ & $\begin{array}{l}\text { LMW heparin } 12 \mathrm{~h} \text { before and for } 4 \text { weeks after } \\
\text { surgery }\end{array}$ \\
\hline \multirow[t]{5}{*}{$\begin{array}{l}\text { Intraoperative } \\
\text { management }\end{array}$} & $\begin{array}{l}\text { Balanced intravenous } \\
\text { fluids }\end{array}$ & Decided by treating anesthetist & Avoid fluid overload \\
\hline & Anesthesia extubating & $\begin{array}{l}\text { Extubating in operating room at operating } \\
\text { team's discretion, routine intensive care } \\
\text { admission if intubated }\end{array}$ & $\begin{array}{l}\text { Extubating in operating room, observation in post- } \\
\text { anesthetic care unit for } 1 \text { hour, then transfer to the } \\
\text { ward }\end{array}$ \\
\hline & $\begin{array}{l}\text { Temperature } \\
\text { management }\end{array}$ & No temperature management & $\begin{array}{l}\text { Active warming with air blanket, maintaining } \\
\text { normothermia }\end{array}$ \\
\hline & PONV prophylaxis & Not used routinely & Triperidol and ondansetron or betamethasone \\
\hline & Abdominal drains & Abdominal drainage at surgeon's discretion & No routine nasogastric or abdominal drainage \\
\hline \multirow[t]{6}{*}{$\begin{array}{l}\text { Postoperative } \\
\text { management }\end{array}$} & Postoperative nutrition & $\begin{array}{l}\text { Clear fluids starting after flatus, daily } \\
\text { progression to diet as tolerated }\end{array}$ & $\begin{array}{l}\text { Free oral drinks } 4 \mathrm{~h} \text { after surgery; free fluids on POD 1; } \\
\text { light meals on POD 2; normal diet from POD } 3\end{array}$ \\
\hline & Mobility exercise & No structured mobilization plans & $\begin{array}{l}\text { Out of bed for at least } 2 \text { hours on day of surgery; at } \\
\text { least } 8 \text { hours, out of bed from POD } 1\end{array}$ \\
\hline & Urethral catheter & Removed at discretion of surgeon & Urethral catheter removed on POD $1 / 3$ \\
\hline & $\begin{array}{l}\text { Postoperative } \\
\text { analgesia and pain } \\
\text { management }\end{array}$ & $\begin{array}{l}\text { Thoracic epidural analgesia or patient- } \\
\text { controlled analgesia until resumption of oral } \\
\text { intake and then transition to oral analgesia }\end{array}$ & $\begin{array}{l}\text { Thoracic epidural analgesia or patient-controlled } \\
\text { analgesia until removal of chest tube, and then } \\
\text { transition to multimodal analgesia (minimizing opioids) }\end{array}$ \\
\hline & Glycemic control & Not used routinely & Insulin protocol in the event of hyperglycemia \\
\hline & Systematic laxatives & No systematic laxatives & Oral magnesium hydroxide \\
\hline
\end{tabular}

CC, conventional care; ERP, enhanced recovery pathway; LMW, low molecular weight; DVT, deep vein thrombosis; PONV, post-operative nausea and vomiting; POD, postoperative day. 
frequencies of each resource consumed were recorded, and multiplied by their respective unit cost to generate the total medical costs. Most of the data came from the front page of medical records, unit costs were supplied by the hospital finance and information management center. Overhead costs like (administration, ERP projects, housekeeping, etc.) were distributed and included.

The real costs were divided into intraoperative and preoperative/postoperative costs. Of note, this study calculated the self-payment that should be borne by patients under the Chinese medical insurance system. They were real costs and not estimated values. The costs of other nonoperative procedures (for example drainage and endoscopy) were included in the general medical services. Logistics costs like housing and administration were counted per day or per admission according to actual costs allocation. The social burdens mainly comprise the patients' lost days from work and the caregiver burden.

Caregiver burden was estimated by asking the patient how long the assistance was required and converted to the average hourly wage in Shanghai. The salary of patients who were not retired were assigned the Shanghai median salary for the analysis. All productivity losses were calculated from a societal perspective using the human capital approach.

A validated method called deviation-based cost modelling was used to analyze the cost effectiveness of the ERPs, which was designed for comparing the clinical and economic impact of clinical pathways (22). Overall expected cost savings refer to the difference in the mean costs between the CC and ERP groups. The effect of the ERP on resource utilization other than hospital bed-days was also investigated.

\section{Statistical analysis}

Univariable comparisons between the CC and ERP groups were performed using $\chi^{2}$ or Fisher's exact test for categorical variables, and Student's $t$-test or Mann-Whitney U test for continuous data. Owing to the extreme skewedness of cost data, $95 \%$ confidence intervals (CIs) around the mean differences between groups were derived from bootstrap estimates (10,000 iterations) taken at the 2.5 th and 97.5 th percentiles. One-way sensitivity analyses were performed by varying number of patients across a wide range of values in the ERP group only (while keeping costs constant in the CC group), and examining the effect on the weighted mean costs. The additional costs were included (such as the cost of the preoperative clinic, potential learning curve differences in operative management over times). All statistical analyses were performed using Stata and SPSS.

\section{Results}

\section{Baseline characteristics}

A total of 106 patients (53 for each group) were included for the analysis. Missing data were not included in the potentially eligible patients. There were no differences in patient and operative characteristics, except for a higher proportion of patients undergoing a retroperitoneal procedure $(\mathrm{P}=0.05)$ and partial adrenalectomy $(\mathrm{P}=0.012)$, as well as fewer having a family history in the ERP group $(\mathrm{P}=0.047)$. Besides, the tobacco smoking and alcohol drinking habits are different between the two groups $(\mathrm{P}=0.022$ and $\mathrm{P}=0.013$, respectively) (Table 2). Although some patient-centered elements were already use in the CC group, the ERP group showed a better compliance. The mean rate of adherence to the protocol was $85 \%$ in the ERP group compared with $46 \%$ in the CC group.

\section{Perioperative outcomes}

Clinical outcomes are reported in Table 3. Duration of operation and anesthesia time were shorter in the ERP group, but showing no differences in either duration time $(\mathrm{P}=0.356$ and $\mathrm{P}=0.435$, respectively). There were significant differences in the postoperative outcomes, such as the LOS was shorter in the ERP group (2.54 days, $\mathrm{P}<0.001)$. The time for patients to leave bed, first anal exhaust, feed, get urethral catheter and drainage tubes removed were all shorter in the ERP group than the CC group $(\mathrm{P}<0.001$ for all). Postoperative complications in both groups were only mild bleeding and infection, for one patient in the ERP group and seven patients in the CC group $(\mathrm{P}=0.031)$, no death occurred.

\section{Cost analysis}

The mean costs for each item are shown in Table 4. The mean \pm standard deviation $(\mathrm{SD})$ intraoperative costs per patient were $¥ 12,532.32 \pm 2,320.69$ for the ERP group and $¥ 13,971.95 \pm 2,356.04$ for the CC group ( $\mathrm{P}=0.002)$. The ERP group was associated with significantly lower treatment disposable materials costs, as well as anesthesia and operating room burden was decreased compared with CC $(\mathrm{P}<0.001)$. Preoperative and postoperative costs 
Table 2 Comparison of patient and operative characteristics between CC and ERP groups

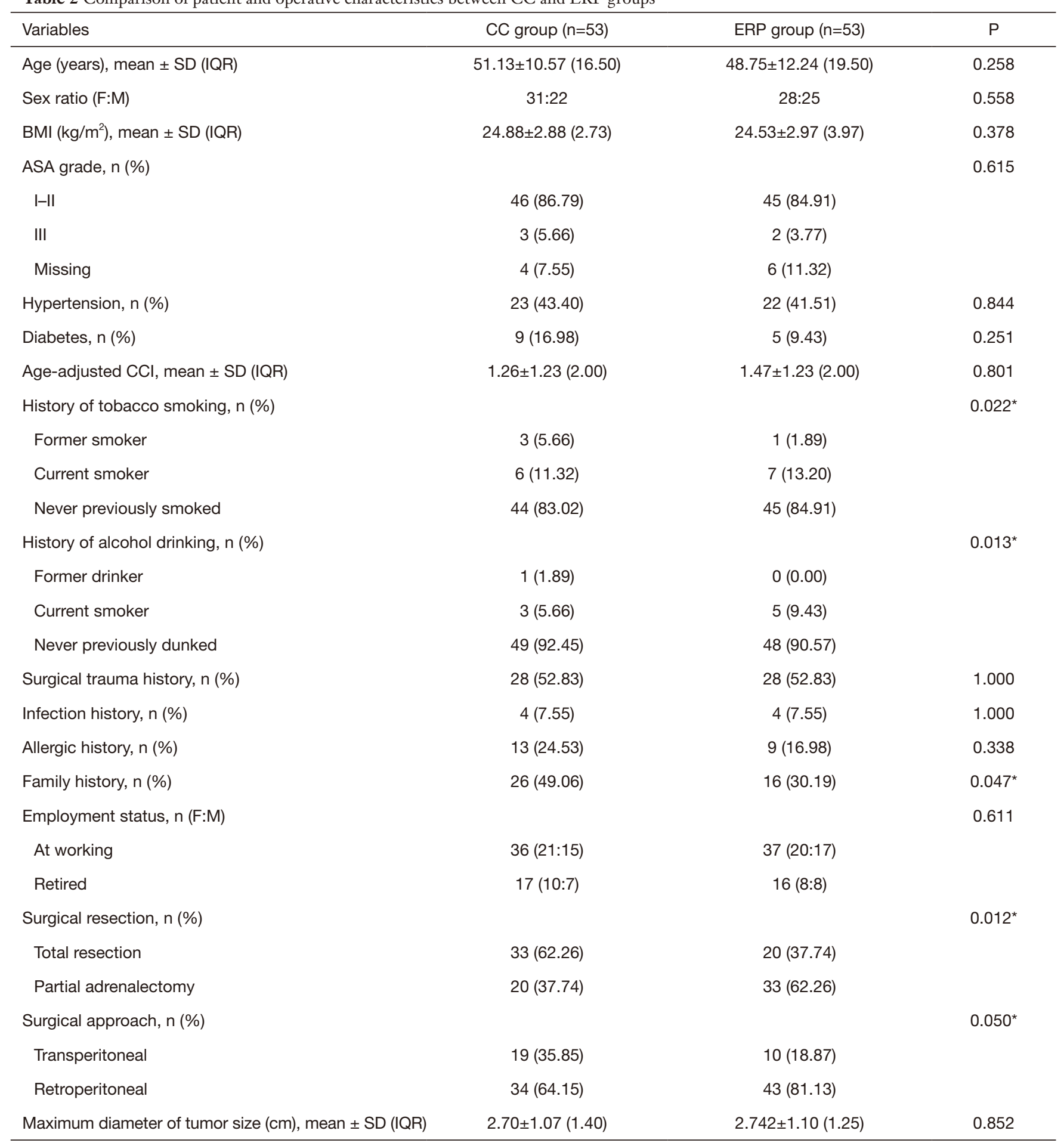

Data are presented as mean $\pm \mathrm{SD}, \mathrm{n}(\%)$ or ratio. CC group: patients receiving CC; ERP group: patients receiving multidisciplinary enhanced pathways after surgery. *, $\mathrm{P}<0.05$. CC, conventional care; ERP, enhanced recovery pathway; SD, standard deviation; IQR, interquartile range; F:M, female: male; BMI, body mass index; ASA, American Society of Anesthesiologist; CCl, Carlson comorbidity index. 
Table 3 Comparison of clinical outcomes between patients managed by CC and ERP groups

\begin{tabular}{|c|c|c|c|}
\hline Variables & CC group $(n=53)$ & ERP group $(n=53)$ & $P$ \\
\hline Duration of operation (min), mean $\pm \mathrm{SD}(\mathrm{IQR})$ & $77.98 \pm 24.09(30.00)$ & $74.91 \pm 26.01(28.00)$ & 0.356 \\
\hline Duration of anesthesia (min), mean $\pm \mathrm{SD}(\mathrm{IQR})$ & $96.85 \pm 24.84(28.75)$ & $93.53 \pm 26.64(30.00)$ & 0.435 \\
\hline \multicolumn{4}{|l|}{ Postoperative outcomes } \\
\hline First anal exhaust time (hour), mean $\pm \mathrm{SD}(\mathrm{IQR})$ & $30.25 \pm 9.63(9.75)$ & $22.76 \pm 10.84(10.25)$ & $<0.001^{\star \star \star}$ \\
\hline Feeding time (hour), mean $\pm \mathrm{SD}$ (IQR) & $32.62 \pm 6.77(7.70)$ & $25.48 \pm 8.53(8.85)$ & $<0.001^{\star \star \star}$ \\
\hline Urethral catheter removal time (hour), mean $\pm \mathrm{SD}(\mathrm{IQR})$ & $35.14 \pm 8.22(8.15)$ & $16.39 \pm 9.74(10.00)$ & $<0.001^{\star \star *}$ \\
\hline Drainage tubes removal time (hour), mean $\pm \mathrm{SD}(\mathrm{IQR})$ & $70.26 \pm 12.35(11.95)$ & $50.42 \pm 14.43(12.15)$ & $<0.001^{\star \star \star}$ \\
\hline \multicolumn{4}{|l|}{ Post discharge outcomes } \\
\hline Lost days from work, mean $\pm \mathrm{SD}(\mathrm{IQR})$ & $16.3 \pm 3.8(4.25)$ & $13.7 \pm 4.2(4.15)$ & $0.003^{* *}$ \\
\hline Caregiver work days, mean $\pm \mathrm{SD}(\mathrm{IQR})$ & $3.2 \pm 1.4(1.37)$ & $1.8 \pm 1.2(1.13)$ & $0.010^{\star \star}$ \\
\hline Postoperative surgeon follow-up visits, mean $\pm S D(I Q R)$ & $1.6 \pm 0.74(0.55)$ & $1.5 \pm 0.46(0.45)$ & 0.653 \\
\hline
\end{tabular}

Data are presented as mean $\pm \mathrm{SD}, \mathrm{n}(\%)$. CC group: patients receiving CC; ERP group: patients receiving multidisciplinary enhanced pathways after surgery. ${ }^{*}, \mathrm{P}<0.05 ;{ }^{* \star}, \mathrm{P}<0.01 ;{ }^{* \star *}, \mathrm{P}<0.001$. CC, conventional care; ERP, enhanced recovery pathway; SD, standard deviation; IQR, interquartile range.

per patient were $¥ 7,469.05 \pm 2,196.02$ in the ERP group and $¥ 14,355.42 \pm 5,952.45$ in the CC group $(\mathrm{P}<0.001)$. ERP was associated with lower costs for preoperative and postoperative costs for nearly all the items in the preoperative and postoperative period (the $\mathrm{P}$ values for inspection disposable materials, surgical disposable materials, surgical operation and traditional Chinese medicine (TCM) were $0.208,0.057,0.318$ and 0.410 respectively, which were not statistically significant; while the $\mathrm{P}$ values for other items were all significant).

\section{Social burden}

The legal retirement age is 60 years old for male and 55 years old for female in China, there were 33 retired patients in this study (Table 2). According to Shanghai Human Resources Security Bureau, the average salary per month (upper and lower limits) is $¥ 10,338(5,975,31,014)$ (23), and the average price for care givers per day is around $¥ 300$. Each month is assumed to be 30 days. The total mean social burden difference per patient was $¥ 1,453.80$ (Table 5). Patients in ERP group was associated with faster return to work and reduced caregiver burden.

\section{Cost-minimum analysis}

The self-payment cost difference per patient between the two groups was $¥ 3,154.52$ in favor of the ERP group, but this was not statistically significant $(\mathrm{P}=0.113)$. The total mean cost difference per patient was $¥ 8,186.53$ in favor of the ERP group $(\mathrm{P}<0.001)$ (Table 4).

The enhanced recovery program execution costs are allocated into services according to the operational items like performance bonuses for participants, the opportunity cost of collaboration time and the costs of patient materials' compilation and printing. In ERAS-specific expenditure, fixed costs were the performance bonus of the ERASdedicated physical therapist ( $¥ 8,500$ per year), crude salary of the ERAS-dedicated nurse ( $¥ 6,000$ per year), the costs of the quarterly ERAS meetings ( $¥ 180$ per meeting), and the costs of publicity pamphlets for patient education ( $¥ 6,353$ per year, including writing time and printing costs). The salary and printing expenses were multiplied by 1.5 , as the study period for the ERAS protocol was 1.5 years. Fixed ERAS costs per patient were therefore $¥ 610.75$ (14,500 $\times$ $1.5 / 53+1,080 / 53+9,529.5 / 53)$. Variable ERAS costs were the costs of the ERAS patient carbohydrate drinks ( $¥ 35$ per 


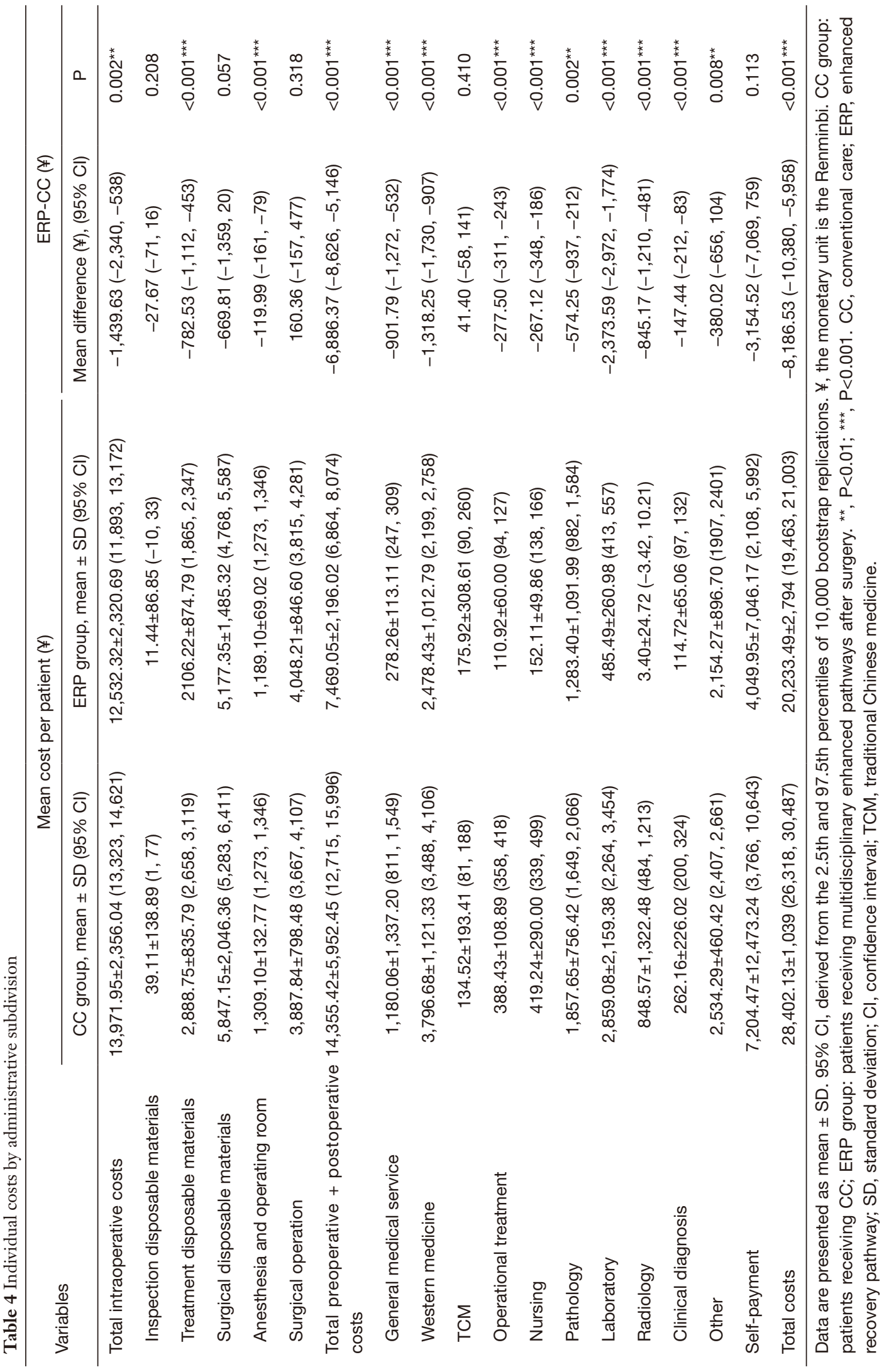


Table 5 Cost-minimization analysis

\begin{tabular}{lccc}
\hline \multirow{2}{*}{ Variables } & \multicolumn{3}{c}{ Mean cost per patient $(¥)$} \\
\cline { 2 - 4 } & CC group $(\mathrm{n}=53)$ & ERP group $(\mathrm{n}=53)$ & Mean difference (ERP-CC) \\
\hline Indirect and potential overhead & $7,335.10$ & $6,537.30$ & -797.80 \\
Social burden (estimated value) & $7,335.10$ & $5,881.30$ & $-1,453.80$ \\
ERAS-specific costs & 0 & 656.00 & 656.00 \\
Explicit overhead & $28,327.37$ & $20,001.37$ & $-8,326.00$ \\
Intraoperative costs & $13,971.95$ & $12,532.32$ & $-1,439.63$ \\
Preoperative and postoperative costs & $14,355.42$ & $7,469.05$ & $-6,886.37$ \\
Total costs & $35,662.47$ & $26,538.67$ & $-18,247.60$ \\
\hline
\end{tabular}

Data are presented as mean. $¥$, the monetary unit is the Renminbi. CC group: patients receiving CC; ERP group: patients receiving multidisciplinary enhanced pathways after surgery. The social burden is an estimated value according to the Shanghai median salary [2020]. CC, conventional care; ERP, enhanced recovery pathway; ERAS, enhanced recovery after surgery.

patient) and warm blanket preheating and holding (¥10 per patient). ERAS-specific costs were thus calculated to be $¥ 656$ per patient. The direct gain per patient with regards to ERAS program was around $¥ 8,326.00$, and the total cost after including potential burden was $¥ 18,247.60$ in favor of the ERP group (Table 5).

\section{Sensitivity analysis}

Some of the ERP costs were divided by the number of patients, therefore the effect of changing the number of patients was examined. If the number was decreased by $50 \%$ (to 26 patients), the mean ERAS-specific cost per patient would be $¥ 1,291.00$, whereas if the number was increased by $50 \%$ (to 79 patients) it would be $¥ 455.75$. The potential gain per patient would be $¥ 554.17$ and $¥ 2,187.22$ for 26 and 79 patients treated according to the ERP respectively.

\section{Discussion}

This study offers insight into the real costs and economic benefits of an enhanced recovery program for laparoscopic adrenalectomy. Standardization perioperative service, controlled materials and medicine, and a significantly shortened hospital stay contributed to this economic benefit. Moreover, this study found ERP had a significant impact on reducing the social burden as less lost days from work and shorter time to hire a rehab caregiver. The present study included all direct costs related to perioperative management as well as all implementation costs of the ERP, including characteristic Chinese traditional medicine costs and overhead cost distribution. Therefore, this study avoided the criticisms of cost analysis studies that lacking complete cost data to the greatest extent (24), savings and losses per patient were in detailed depicted.

The significant reduction in complication rate $(\mathrm{P}=0.031)$ and LOS $(\mathrm{P}<0.001)$ might explain the cost reduction observed, as has been considered the mainly common ways influencing the costs $(25,26)$. However, the duration of hospital stay has a limited impact on overall costs for its inequivalent economically, the first few postoperative days (PODs) are usually more costly than the days before discharge (27). Some studies showed the postoperative complications had a major impact on costs, especially for those surgery with high risk, the complications and severity were important to hospital charges (28). In the present study, the complications were not server, only mild bleeding and infection were found and treated. No patients required a readmission to hospital and no tumor recurrence was found in the study period. Therefore, though complications in the ERP group were less severe, economic impact on complications was proved. The average age of the two groups were $51.13 \pm 10.57$ and $48.75 \pm 12.24$, respectively. Although elder patient factors contribute to adverse events and increased costs (29), standardized surgical care pathways can improve the quality and efficiency of care (30). No severe complications or medical death occurred in the present study.

Similar effective results have been found in other urology procedures like bladder and prostate surgery. ERPs effectively accelerate patient rehabilitation and reduce LOS and hospitalization costs for prostate cancer patients undergoing laparoscopic radical prostatectomy (31), 
prehabilitation exercise was integrated in the ERPs. Moreover, prehabilitation pathway along with ERPs and robotic surgery has also been suggested to work synergistically in improving outcomes after inpatient robotassisted radical prostatectomy (RARP), and to gain a wider acceptance of same day discharge (SDD), which is supposed to have a positive impact on pain control, general activity, and reduced overall costs. A multi-surgeon comparative study confirms the safety of routine SDD and RARP in terms of perioperative outcomes (32), trends favoring ERPs combined with prehabilitation, SSD and robotic surgery need to be confirmed, these improvements may promote high quality of life together for patients. Another two studies provided available data to support ERPs for patients undergoing radical cystectomy leading to a shorter LOS without increasing readmission or complication rates $(33,34)$, suggesting more solid evidence of assessing the clinical and economic impact of ERPs.

There have been no cost-minimization studies of ERP in adrenal mass surgery, available comparable data are sparse. Two previous studies, which did not involve potential social burdens but include implementation costs, reported similar significant savings following the implementation of ERP for colorectal surgery and pancreaticoduodenectomy respectively $(17,18)$. A cost-effectiveness analysis of perioperative management for colorectal surgery took overall societal costs into account and found that patients in the ERP group returned to work more quickly and had less caregiver burden (35), which was consistent with this study. However, loss of productivity was not accounted. There was no study on adrenal surgery with detailed costminimization analysis and the analysis on this disease were not considered potential costs as usual. In contrast, the present study proved the cost-effectiveness of ERAS for laparoscopic adrenalectomy, found the cost savings associated with ERPs with Chinese characteristics [such as the TCM use and the per-capita wage], and may further encourage ERAS adoption in China being the optimal perioperative management approach. TCM is considered to be a safe traditional way with less stress or side effects on human body, which is in line with the ERAS concept of Chinese characteristics. The TCM costs were a little more in the ERP group but showed no significance compared with the CC group $(\mathrm{P}=0.41)$. The ERP was associated with significantly fewer total costs, as perioperative burden was significantly decreased compared with the CC group.

The present study has some limitations. First, there were differences in the surgical approaches between the two groups. Adrenal tumor is a disease that differs from other tumors in many ways. The adrenal gland has a unique function that can secrete a variety of important hormones, the more tissue was removed, the more endocrine function of the gland was likely to be affected. In addition, the location of adrenal tumor is also relatively special, surrounded by large vessels and important organs. In laparoscopic surgery, it is more difficult to preserve part of the adrenal gland than to perform total resection. This study controlled the size of adrenal tumors within a reasonable range, but the difficulty of the operation still varied, which may affect the duration and cost of the operation. Second, the study was implemented in the regional central hospital in Shanghai, there may patients coming from other provinces and cities. The costs of follow-ups in different regions and transfer of costs from the hospital to the community were not counted, and the transportation costs were not evaluated in the present study, either. Third, this study included a matched casecontrol study, which is non-random. Patients diagnosed with adrenal mass and treated with ERPs were used as cases and comparable individuals treated with CC were used as controls. It may introduce selection biases between although the control of demographics and tumor parameters reduced that risk. After data screening, the small sample size (106 patients) limits generalizability, though our results were corroborated by prior studies in other diseases like colorectal surgery. Finally, the different subgroup analysis of economic results needs further investigation, such as patients undergoing surgery using transperitoneal and retroperitoneal approaches may have cost differences, and a recent study showed that posterior retroperitoneoscopic adrenalectomy could result in better clinical outcomes (36). In addition, the excluded subgroups of pheocromocytoma and Cushing syndrome should carry out cost-effectiveness analysis under the respective diagnosis and treatment pathways. Hospital administrations and healthcare purchasers require information about cost when deciding whether to adopt new quality initiatives like ERPs, and data have been lacking to support the touted economic benefits of ERPs for complex procedures by rigorous evaluation (18). ERPs associated with reduced costs by virtue of reducing individual expenses need more available evidence.

\section{Conclusions}

ERPs were appropriate interventions to improve clinical and economic outcomes, which resulted in lower overall 
cost savings and reduced social burdens than conventional management in patients undergoing laparoscopic adrenalectomy. This study provides further evidence to support the adoption of ERPs in China, reducing risks and burdens associated with adrenal operations.

\section{Acknowledgments}

The authors thank the hospital for providing the data, and all members of the urological surgery team and ERAS team at this institution for their daily clinical and research work. Funding: This study was funded by grant from Shanghai Municipal Natural Science Foundation (No. 19ZR1431800).

\section{Footnote}

Reporting Checklist: The authors have completed the TREND reporting checklist. Available at https:// gs.amegroups.com/article/view/10.21037/gs-21-504/rc

Data Sharing Statement: Available at https://gs.amegroups. com/article/view/10.21037/gs-21-504/dss

Conflicts of Interest: All authors have completed the ICMJE uniform disclosure form (available at https://gs.amegroups. com/article/view/10.21037/gs-21-504/coif). The authors have no conflicts of interest to declare.

Ethical Statement: The authors are accountable for all aspects of the work in ensuring that questions related to the accuracy or integrity of any part of the work are appropriately investigated and resolved. The study was conducted in accordance with the Declaration of Helsinki (as revised in 2013). The study was approved by the Ethics Committee of the Ruijin Hospital, Shanghai Jiao Tong University School of Medicine (No. 2020-00648), and informed consent was taken from all individual participants.

Open Access Statement: This is an Open Access article distributed in accordance with the Creative Commons Attribution-NonCommercial-NoDerivs 4.0 International License (CC BY-NC-ND 4.0), which permits the noncommercial replication and distribution of the article with the strict proviso that no changes or edits are made and the original work is properly cited (including links to both the formal publication through the relevant DOI and the license). See: https://creativecommons.org/licenses/by-nc-nd/4.0/.

\section{References}

1. Raffaelli M, De Crea C, Bellantone R. Laparoscopic adrenalectomy. Gland Surg 2019;8:S41-52.

2. Torti JF, Correa R. Adrenal Cancer. In: StatPearls. Treasure Island: StatPearls Publishing LLC, 2021.

3. Cingam SR, Mukkamalla SKR, Karanchi H. Adrenal Metastasis. In: StatPearls. Treasure Island: StatPearls Publishing LLC, 2021.

4. Forsmo HM, Pfeffer F, Rasdal A, et al. Pre- and postoperative stoma education and guidance within an enhanced recovery after surgery (ERAS) programme reduces length of hospital stay in colorectal surgery. Int J Surg 2016;36:121-6.

5. Sun Y, Li Z, Liu X, et al. A Nomogram for Prediction of Survival in Patients After Gastrectomy Within Enhanced Recovery After Surgery (ERAS): A Single-Center Retrospective Study. Med Sci Monit 2020;26:e926347.

6. Qu L, Liu B, Zhang H, et al. Management of Postoperative Pain after Elective Craniotomy: A Prospective Randomized Controlled Trial of a Neurosurgical Enhanced Recovery after Surgery (ERAS) Program. Int J Med Sci 2020;17:1541-9.

7. Li Q, Wan L, Liu S, et al. Clinical efficacy of enhanced recovery after surgery in percutaneous nephrolithotripsy: a randomized controlled trial. BMC Urol 2020;20:162.

8. Cerantola Y, Valerio M, Persson B, et al. Guidelines for perioperative care after radical cystectomy for bladder cancer: Enhanced Recovery After Surgery (ERAS(®)) society recommendations. Clin Nutr 2013;32:879-87.

9. Vlad O, Catalin B, Mihai H, et al. Enhanced recovery after surgery (ERAS) protocols in patients undergoing radical cystectomy with ileal urinary diversions: A randomized controlled trial. Medicine (Baltimore) 2020;99:e20902.

10. Lin T, Li K, Liu H, et al. Enhanced recovery after surgery for radical cystectomy with ileal urinary diversion: a multi-institutional, randomized, controlled trial from the Chinese bladder cancer consortium. World J Urol 2018;36:41-50.

11. Petersen RH, Huang L, Kehlet H. Guidelines for enhanced recovery after lung surgery: need for re-analysis. Eur J Cardiothorac Surg 2021;59:291-2.

12. Ljungqvist $O, S \operatorname{Scott} M$, Fearon KC. Enhanced Recovery After Surgery: A Review. JAMA Surg 2017;152:292-8.

13. Roulin D, Blanc C, Muradbegovic M, et al. Enhanced recovery pathway for urgent colectomy. World J Surg 2014;38:2153-9.

14. National Health Commission of the People's Republic of 
China. 2020 Statistical Communique on the Development of China's Health Undertakings. 2021. Available online: http://www.nhc.gov.cn/guihuaxxs/s10743/202107/af8a9c9 8453c4d9593e07895ae0493c8.shtml

15. Chai S, Pan Q, Liang C, et al. Should surgical drainage after lateral transperitoneal laparoscopic adrenalectomy be routine?-A retrospective comparative study. Gland Surg 2021;10:1910-19.

16. Maestroni U, Cataldo S, Moretti V, et al. A challenging case of laparoscopic synchronous bilateral adrenalectomy for Cushing's disease. Case report. Ann Med Surg (Lond) 2018;36:261-3.

17. Joliat GR, Labgaa I, Petermann D, et al. Costbenefit analysis of an enhanced recovery protocol for pancreaticoduodenectomy. Br J Surg 2015;102:1676-83.

18. Lee L, Mata J, Ghitulescu GA, et al. Cost-effectiveness of Enhanced Recovery Versus Conventional Perioperative Management for Colorectal Surgery. Ann Surg 2015;262:1026-33.

19. Koppie TM, Serio AM, Vickers AJ, et al. Ageadjusted Charlson comorbidity score is associated with treatment decisions and clinical outcomes for patients undergoing radical cystectomy for bladder cancer. Cancer 2008;112:2384-92.

20. Charlson M, Szatrowski TP, Peterson J, et al. Validation of a combined comorbidity index. J Clin Epidemiol 1994;47:1245-51.

21. Clavien PA, Barkun J, de Oliveira ML, et al. The ClavienDindo classification of surgical complications: five-year experience. Ann Surg 2009;250:187-96.

22. Vanounou T, Pratt W, Fischer JE, et al. Deviation-based cost modeling: a novel model to evaluate the clinical and economic impact of clinical pathways. J Am Coll Surg 2007;204:570-9.

23. Shanghai Human Resources and Social Security Bureau. Announcement on the 2020 average wage of employed persons in urban. 2021. Available online: https:// mp.weixin.qq.com/s/re3XWWE1iwEzSmqsze9fkg

24. Roulin D, Donadini A, Gander S, et al. Cost-effectiveness of the implementation of an enhanced recovery protocol for colorectal surgery. Br J Surg 2013;100:1108-14.

25. Attrill S, White S, Murray J, et al. Impact of oropharyngeal dysphagia on healthcare cost and length of stay in hospital: a systematic review. BMC Health Serv Res 2018;18:594.

26. Chin-Yee N, D'Egidio G, Thavorn K, et al. Cost analysis of the very elderly admitted to intensive care units. Crit Care 2017;21:109.

27. Taheri PA, Butz DA, Greenfield LJ. Length of stay has minimal impact on the cost of hospital admission. J Am Coll Surg 2000;191:123-30.

28. De la Plaza Llamas R, Ramia JM. Cost of postoperative complications: How to avoid calculation errors. World J Gastroenterol 2020;26:2682-90.

29. Davenport DL, Henderson WG, Khuri SF, et al. Preoperative risk factors and surgical complexity are more predictive of costs than postoperative complications: a case study using the National Surgical Quality Improvement Program (NSQIP) database. Ann Surg 2005;242:463-8; discussion 468-71.

30. Lee L, Li C, Robert N, et al. Economic impact of an enhanced recovery pathway for oesophagectomy. Br J Surg 2013;100:1326-34.

31. Lin C, Wan F, Lu Y, et al. Enhanced recovery after surgery protocol for prostate cancer patients undergoing laparoscopic radical prostatectomy. J Int Med Res 2019;47:114-21.

32. Rahota RG, Salin A, Gautier JR, et al. Same Day Discharge versus Inpatient Surgery for Robot-Assisted Radical Prostatectomy: A Comparative Study. J Clin Med 2021;10:661.

33. Pozo C, Shariat SF, D'Andrea D, et al. Enhanced Recovery after Radical Cystectomy. Curr Opin Urol 2019;29:227-38.

34. Frees SK, Aning J, Black P, et al. A prospective randomized pilot study evaluating an ERAS protocol versus a standard protocol for patients treated with radical cystectomy and urinary diversion for bladder cancer. World J Urol 2018;36:215-20.

35. Lee L, Li C, Landry T, et al. A systematic review of economic evaluations of enhanced recovery pathways for colorectal surgery. Ann Surg 2014;259:670-6.

36. Kook Y, Choi HR, Kang SW, et al. Laparoscopic adrenalectomy: comparison of outcomes between posterior retroperitoneoscopic and transperitoneal adrenalectomy with 10 years' experience. Gland Surg 2021;10:2104-12.

Cite this article as: Yan Y, Cheng J, Chen K, Liu TF, Ning G. Better clinical benefits and potential cost saving of an enhanced recovery pathways for laparoscopic adrenalectomy. Gland Surg 2022;11(1):23-34. doi: 10.21037/gs-21-504 